\title{
Infecção de loja de cardioversor-desfibrilador implantável (CDI) por klebsiella sp. Carbapenem- resistente
}

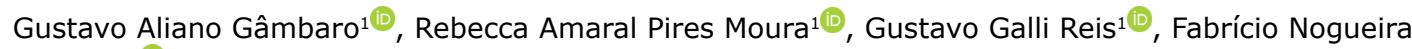
Furtado 2 (1)

\begin{abstract}
RESUMO
As infecções associadas aos Dispositivos Cardíacos Eletrônicos Implantáveis (DCEI) apresentam uma incidência de até $3,4 \%$ e notável impacto na morbidade e mortalidade dos pacientes. As bactérias Gram-positivas, especialmente do gênero Staphylococcus sp. representam 60-70\% dos agentes isolados. Por sua vez, as Gram-negativas correspondem até $9 \%$ dos casos. Relatamos uma infecção de loja de gerador de Cardioversor-desfibrilador implantável (CDI) por uma Klebsiella sp. resistente aos carbapenêmicos, em um paciente masculino jovem, cujo desafiador diagnóstico de certeza desse caso somente foi possível após exploração cirúrgica e cultura do material da loja do CDI, haja vista a apresentação clínica oligossintomática. Embora já descritas, Klebsiella sp. são raras nesse contexto e em nosso conhecimento, esse é o primeiro relato de uma infecção de DCEI por uma enterobactéria resistente a carbapenêmico.

Palavras-chave: Desfibriladores implantáveis, Enterobacteriáceas resistentes a carbapenêmicos, Infecções por Klebsiella.
\end{abstract}

1. Pontifícia Universidade Católica do Paraná, campus Londrina. Escola de Medicina

2. Universidade Estadual de Londrina 


\section{INTRODUÇÃO}

A taxa de implantes de dispositivos cardíacos eletrônicos implantáveis (DCEI) aumentou consideravelmente em todo o mundo e, aproximadamente, 1,5 milhão de pacientes recebem DCEI anualmente ${ }^{1}$. Contudo, infecções associadas aos dispositivos apresentam uma incidência de até 3,4\% e acarretam notável impacto na morbidade assim como na mortalidade, podendo atingir até $8 \%$ em 30 dias ${ }^{2}$.

O principal mecanismo fisiopatológico dessa complicação é a contaminação dos fios eletrodos e/ou do gerador durante o implante ou manipulação subsequente. A colonização do material da loja do gerador pode se propagar através dos cabos eletrodos e resultar em infecção sistêmica. Disseminação hematogênica a partir de outros focos infecciosos também foi descrita ${ }^{2,3}$.

As bactérias Gram-positivas são os agentes mais importantes dessas infecções, dos quais $36,7 \%$ correspondem a Staphylococcus Coagulasenegativo e $30,8 \%$ ao Staphylococcus aureus ${ }^{2-5}$. Os agentes gram-negativos perfazem cerca de 6-9\% dos isolados, e pertencem ao gênero Enterobacteriaceae em até $3 \%$ dos casos $2,3,5$. De similar modo, infecções fúngicas e micobacterianas foram descritas ${ }^{2-6}$.

Relatamos uma infecção de loja de gerador de um Cardioversor-Desfibrilador Implantável Transvenoso (CDI-T), cujo agente isolado na cultura foi uma Klebsiella sp. resistente aos carbapenêmicos. Nesse caso, houve significativo desafio diagnóstico em vista da apresentação clínica oligossintomática e da precocidade das manifestações.

\section{RELATO DO CASO}

Um jovem de 21 anos, sexo masculino, acadêmico de medicina, apresentou sangramento persistente da ferida operatória a partir do quarto dia de pós-operatório de implante eletivo de CDI-T, devido ao diagnóstico de cardiopatia geneticamente determinada por mutação da desmoplaquina (DSP). O paciente negava outros sinais e sintomas, incluindo febre. Durante a indução anestésica para profilaxia antimicrobiana, a Cefazolina 2 gramas havia sido administrada. Ele estava em uso de Prednisona 20 miligramas diariamente, por diagnóstico anterior de Miopericardite recorrente.

Ao exame clínico, a ferida operatória não apresentava sinais flogísticos ou drenagem de secreção purulenta. Contudo, havia discreta deiscência das bordas lateralmente. Não havia eritema, edema ou equimoses na pele adjacente à loja do gerador (Figura 1). Ademais, o exame físico não revelou quaisquer alterações. Portanto, foi optado por conduta conservadora e vigilância clínica.

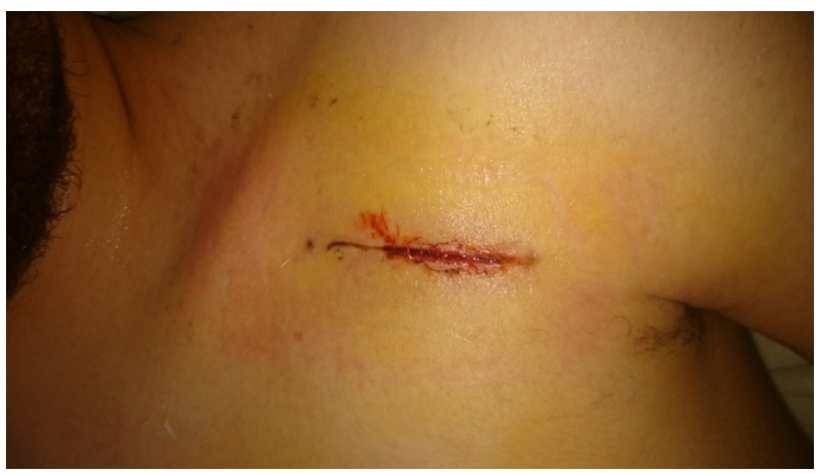

Figura 1. Ferida operatória sem sinais flogísticos, porém, com discreta deiscência em região lateral associada a sangramento. Não se observa drenagem de conteúdo purulento.

Entretanto, após cinco dias de medidas conservadoras, embora o paciente se mantivesse afebril, havia persistência do sangramento e optou-se pela reavaliação cirúrgica. Antes do procedimento, foram colhidas amostras para hemoculturas. A vancomicina $(15 \mathrm{mg} / \mathrm{kg})$ foi administrada como profilaxia e o paciente apresentou eritema maculopapular pruriginoso em pescoço, tórax e extremidades, compatível com Síndrome do Homem Vermelho. O rash cessou com o uso de anti-histamínico.

Durante o intra-operatório, observou-se tecido necrótico e friável na bolsa do gerador e, portanto, diante de suspeita de infecção de loja de gerador, procedeuse com a extração completa do dispositivo e coleta de amostras de tecido da loja. As pontas dos cabos eletrodos do CDI e as amostras de tecido foram enviadas para cultura. O paciente então permaneceu internado, clinicamente estável em uso empírico de Teicoplanina (6 mg/kg/dia), devido a reação à Vancomicina, enquanto aguardava resultado microbiológico.

A cultura da loja evidenciou Klebsiella $s p$. Carbapenem-resistente (CR), sensível somente à gentamicina ao antibiograma (Figura 2). Ambas as amostras de hemocultura e a cultura da ponta dos cabos eletrodos foram negativas. Além disso, não havia vegetações ao Ecocardiograma Transesofágico (ETE) (Figura 3). Desse modo, descartou-se endocardite infecciosa (EI) e a antibioticoterapia foi escalonada para Polimixina B (25.000 UI/kg 12/12h) e Gentamicina ( $5 \mathrm{mg} / \mathrm{kg} / \mathrm{dia}$ ). 
CULTURA BACTERIANA

Material: Loja

RESULTADO: Klebsiella sp

AG. TERAPÊUTICO

SENSIBILIDADE

AG. TRRAPÊUTICO

SENSIBILIDADE

ERTAPENEN............ Resistente

CEFEPIME............ Resistente

AMICACINA. . . . . . . . . Resistente PIPERACILINA TAZOBACTAM..: Resistente

NOTA: Antibióticos testados de acordo com tabela CLSI Atualizada

Data de Coleta: 01/05/2018 às 07:08 - Material entregue ao laboratório.

Código de segurança:cb0f59963cc0ea04eleeco3d994e7813

Data: $06 / 05 / 18 \quad 20: 22$

\author{
CULTURA - $2^{\text {a }}$ amostra \\ Material: Ponta \\ RESULTADO: NEGATIVO
}

Figura 2. Cultura da loja do gerador evidenciou Klebsiella sp. resistente a carbapenêmicos (CR). Cultura da ponta do cabo eletrodo negativa.
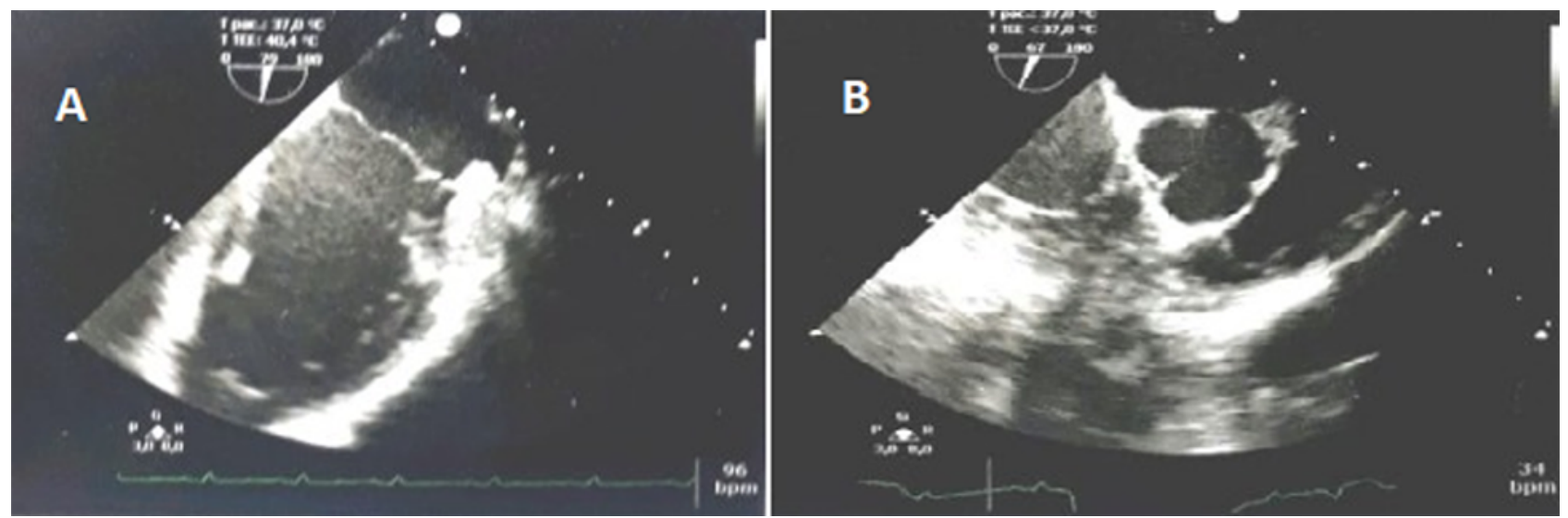

Figura 3. A - Não se observa lesões em valva mitral; B- Valva aórtica e tricúspide íntegras e sem evidência de vegetações. 
No entanto, após infusão da primeira dose de Polimixina B, o paciente apresentou parestesia em face, bilateralmente. Consequentemente, o paciente manifestou forte desejo de suspensão da droga. Após deliberação e esclarecimento ao paciente a respeito dos riscos de monoterapia com Gentamicina, optouse por descontinuação da Polimixina, visto que seu estado clínico era estável.

Esse esquema terapêutico foi administrado por 14 dias e o paciente se manteve em boa evolução clínica. Não foram observadas novas complicações da ferida operatória ou efeitos adversos e o paciente recebeu alta do serviço ao final da segunda semana.

Por fim, três meses depois, o paciente implantou um CDI subcutâneo (CDI-S) e permaneceu livre complicações. Nessa ocasião, foram coletados swabs de pele pré-operatórios, todos negativos, e o paciente foi orientado a realizar banho de clorexidina no pré-operatório.

\section{DISCUSSÃO}

A European Heart Rhythm Association (EHRA) publicou recentemente um consenso ${ }^{2}$ que sumariza as principais recomendações para o diagnóstico, tratamento e prevenção das infecções em DCEI.

No processo de indicação de um DCEI é imperativo avaliar os fatores de risco ${ }^{2,4}$. Esses podem ser modificáveis ou não modificáveis. A Doença Renal Crônica dialítica foi fortemente associada a infecções, a semelhança do uso de corticoesteroides ${ }^{2}$. Em nosso relato, o paciente fazia uso de Prednisona $20 \mathrm{mg}$ por dia, fato que pode ter contribuído para sua complicação.

A maior complexidade do dispositivo, como CDI-T dupla câmara e ressincronizadores, configura outro fator de risco. Finalmente, a maior duração dos procedimentos e a necessidade de reintervenção (hematomas, por exemplo) aumentam as infecções relacionadas ao dispositivo ${ }^{2-6}$.

Clinicamente, a infecção de DCEI apresenta espectro variável desde infecções incisionais superficiais à endocardite infecciosa. As infecções de loja são as mais comuns e se manifestam com edema, eritema, deiscência e secreção no local da loja. Por sua vez, as infecções sistêmicas podem ocorrer na ausência de processo infeccioso da loja do gerador. Esse fato torna o diagnóstico mais desafiador e deve haver alta suspeição quando há febre, calafrios, sudorese noturna e fenômenos embólicos sépticos ${ }^{2-7}$.

O diagnóstico é baseado nos critérios internacionais de infecção de DCEI de 2019, que incluem aspectos microbiológicos, radiológicos e clínicos $^{2}$. Este caso ilustra a dificuldade de diagnóstico de pacientes oligossintomáticos. Recentemente, a tomografia por emissão de pósitrons marcada com flúor-18 (18F-FDG PET-CT) despontou como um promissor método diagnóstico adjuvante. Com destaque para estabelecer o diagnóstico diferencial entre infecções de loja e inflamação pós-cirúrgica, ou quando há alta suspeita de infecção sistêmica, mas o ETE é negativo ${ }^{2,3,8}$.

O ETE é recomendado para avaliação de infecção em cabos eletrodos e EI associada ao DCEI. Após extração do dispositivo, o ETE pode ser considerado, a fim de identificar possíveis complicações da valva tricúspide, função ventricular direita e resíduos do dispostivo ${ }^{2-5}$.

A aspiração com agulha da loja, a fim de estabelecer o agente etiológico, não é recomendada devido à baixa sensibilidade e à possibilidade teórica de contaminação da loja ${ }^{3}$. Por conseguinte, amostras do tecido da loja devem ser coletadas para cultura, assim como as pontas dos cabos eletrodos se a extração do dispositivo é necessária. Recomendase ao menos três amostras de hemocultura se há suspeita de endocardite infecciosa ${ }^{2-6}$.

$\mathrm{O}$ isolamento de patógenos resistentes é preocupante nesse cenário e em $33,8 \%$ das infecções em DCEI, foram isolados Staphylococcus resistentes à Meticilina. Da mesma forma, cepas de Enterococcus resistentes à Vancomicina correspondem a 1,4\% dos agentes isolados na América do Norte ${ }^{2}$. Em relação à etiologia da infecção desse relato, Klebsiella sp. são raras neste contexto, embora descritas previamente ${ }^{9}$. Em nosso conhecimento, este é o primeiro relato de infecção de CDI por Klebsiella sp. resistente a carbapenêmicos.

No serviço onde esse procedimento foi realizado, a taxa de Infecções Relacionadas à Assistência à Saúde (IRAS) no mês do implante foi de $2,7 \%$. Esse índice encontra-se aceitável, considerando a prevalência entre $5,7 \%$ a $19,1 \%$ de IRAS nos países em desenvolvimento segundo a Organização Mundial da Saúde ${ }^{10}$. Ademais, não houve relato de quebra da técnica asséptica ou contaminação do campo cirúrgico no intra-operatório. 
No que se refere ao tratamento, a retirada do dispositivo é fortemente recomendada. A exceção consiste em infecções superficiais da pele, que devem ser tratadas com antibióticos orais, como flucloxacilina ou amoxicilina-clavulanato por pelo menos uma semana ${ }^{2,3}$. Por sua vez, infecções de loja requerem 10-14 dias de terapia antibiótica após a extração. Os regimes de escolha são Vancomicina (ou Daptomicina) isolada ou em associação com cefalosporina de terceira geração ou Gentamicina, em especial se sintomas sistêmicos. Para infecções profundas, como a EI, são necessárias 4-6 semanas de agentes antimicrobianos 2,3; 5-7. $^{2}$.

O envelope antibacteriano libera rifampicina e minociclina e está indicado em situações de maior probabilidade de infecção, tais como reoperação, upgrade de dispositivo, instalação de ressincronizador e de pacientes de alto risco. Contudo, sua capacidade de prevenir infecções por agentes multirresistentes é incerta ${ }^{2,11}$.

Finalmente, os pacientes com extração prévia de CDI-T por infecção podem ser elegíveis para o CDI-S, que não requer eletrodos transvenosos e, portanto, reduz o risco de eventos mais graves como EI e complicações relacionadas aos cabos eletrodos. Nessa população de pacientes, não houve aumento do risco de infecção de novo após reimplante de CDI-S ${ }^{12}$.

Este caso deve alertar para a possibilidade de bactérias multirresistentes como possíveis agentes etiológicos da infecção em DCEI, além da necessidade urgente de métodos adjuvantes para preveni-las e controlar a resistência bacteriana. Enfim, um alto índice de suspeição para infecção é necessário diante de complicações perioperatórias de DCEI para que se estabeleça uma conduta diagnóstica e terapêutica em tempo hábil e melhore o prognóstico do paciente ${ }^{13}$.

\section{REFERÊNCIAS}

1.Mond HG, Proclemer A. The 11th world survey of cardiac pacing and implantable cardioverter-defibrillators: calendar year 2009--a World Society of Arrhythmia's project. Pacing Clin Electrophysiol. 2011;34(8):10131027. doi: $10.1111 / j .1540-8159.2011 .03150 . x$

2.Carina Blomström-Lundqvist, Vassil Traykov, Paola Anna Erba, Haran Burri, Jens Cosedis Nielsen, Maria Grazia Bongiorni, Jeanne Poole, Giuseppe Boriani, Roberto Costa, Jean-Claude Deharo, Laurence M Epstein, László Sághy, Ulrika SnyggMartin, Christoph Starck, Carlo Tascini, Neil Strathmore,
European Heart Rhythm Association (EHRA) international consensus document on how to prevent, diagnose, and treat cardiac implantable electronic device infections-endorsed by the Heart Rhythm Society (HRS), the Asia Pacific Heart Rhythm Society (APHRS), the Latin American Heart Rhythm Society (LAHRS), International Society for Cardiovascular Infectious Diseases (ISCVID), and the European Society of Clinical Microbiology and Infectious Diseases (ESCMID) in collaboration with the European Association for CardioThoracic Surgery (EACTS), European Heart Journal, Volume 41, Issue 21, 1 June 2020, Pages 2012-2032, https://doi. org/10.1093/eurheartj/ehaa010

3.Arnold CJ, Chu VH. Cardiovascular Implantable Electronic Device Infections. Infect Dis Clin North Am. 2018;32(4):811-825. doi:10.1016/j.idc.2018.06.004

4.Uslan DZ, Sohail MR, St Sauver JL, et al. Permanent pacemaker and implantable cardioverter defibrillator infection: a population-based study. Arch Intern Med. 2007;167(7):669-675. doi:10.1001/archinte.167.7.669

5. Harrison JL, Prendergast BD, Sandoe JA. Guidelines for the diagnosis, management and prevention of implantable cardiac electronic device infection. Heart. 2015;101(4):250-252. doi:10.1136/ heartjnl-2014-306873

6.DeSimone DC, Sohail MR, Mulpuru SK. Contemporary management of cardiac implantable electronic device infection. Heart. 2019;105(12):961-965. doi:10.1136/ heartjnl-2017-312146

7.DeSimone DC, Sohail MR. Infection Management. Card Electrophysiol Clin. 2018;10(4):601-607. doi:10.1016/j. ccep.2018.04.002

8.Amraoui S, Tlili G, Sohal M, et al. Contribution of PET Imaging to the Diagnosis of Septic Embolism in Patients With Pacing Lead Endocarditis. JACC Cardiovasc Imaging. 2016;9(3):283-290. doi:10.1016/j.jcmg.2015.09.014

9. Ertas F, Acet $H$, Kaya H, Kayan F, Soydinc S. Implantable cardioverter defibrillator pocket infection caused by Klebsiella pneumonia. Afr Health Sci. 2012;12(3):388-389.

10. WHO. Health care-associated infection. FACT SHEET.

11.Tarakji KG, Mittal S, Kennergren C, et al. Antibacterial Envelope to Prevent Cardiac Implantable Device Infection. N Engl J Med. 2019;380(20):1895-1905. doi:10.1056/NEJMoa1901111

12. Boersma L, Burke MC, Neuzil $P$, et al. Infection and mortality after implantation of a subcutaneous ICD after transvenous ICD extraction. Heart Rhythm. 2016;13(1):157-164. doi:10.1016/j.hrthm.2015.08.039

13. Viganego F, O'Donoghue S, Eldadah Z, et al. Effect of early diagnosis and treatment with percutaneous lead extraction on survival in patients with cardiac device infections. Am J Cardiol. 2012;109(10):1466-1471. doi:10.1016/j.amjcard.2012.01.360 
Infecção de CDI por Enterobactéria multirresistente (MR)

Autor Correspondente:

Gustavo Aliano Gâmbaro

gustavogambaro2@gmail.com

Editor:

Prof. Dr. Paulo Henrique Manso

Recebido: $12 / 09 / 2020$

Aprovado: 12/08/2021 